\title{
Liquid-Crystal Based Light Steering Optical Elements
}

\author{
Sergiy Valyukh ${ }^{1}$, Iryna Valyukh ${ }^{1}$, Vladimir Chigrinov $^{2}$ \\ ${ }^{1}$ Laboratory of Applied Optics, IFM, Linköping University, SE-581 83 Linköping, Sweden \\ ${ }^{2}$ Hong Kong University of Science and Technology, CWB, Kowloon, Hong Kong
}

Received April 17, 2011; accepted June 28, 2011; published June 30, 2011

\begin{abstract}
Potential abilities of active optical elements based on liquid crystals are investigated. We consider liquid crystal cells in which a desired distribution of the liquid crystal director is achieved by a nonuniform electric field and by inhomogeneous boundary conditions. The optical elements built on principles of diffractive optics are discussed.
\end{abstract}

Effective and compact active optical elements having tunable characteristics such as lenses with variable focus distance, beam steerers, and dynamic diffractive gratings are highly required for integration in numerous optoelectronic devices and photonic applications. Liquid crystals (LCs), that possess the ability to be controlled with an external electric field are especially attractive for tunable optical elements. Because of birefringence, the optical path of an LC layer depends on orientations of the LC molecules (director). By generating a spatially nonuniform electric field inside the $\mathrm{LC}$, it is possible to form a distribution of the LC director that causes a distribution of the optical path. A light wave traveling through such an LC layer changes the wavefront. By this means the shape of the wavefront, as well as the direction of the propagation, can be controlled with an electric field applied to the LC cell.

In the present letter, we consider the basic principles of the LC optical elements and evaluate their potential functional abilities.

Fig. 1 shows lays rays passing the beam steering device (a) and collecting lens (b). Both optical elements adapted for transmissive mode. These optical elements differ in profiles of the phase delays formed by them. When the phase delay of an LC cell has a linear dependence versus the space coordinate (Fig. 1 a), such an LC cell, from the optical point of view, is equivalent to a prism. The parabolic profile of the phase delay of the LC cell (Fig. 1 b) causes the lens effect.

The optical path of the LC cell along the $X$ axis can be found according to the following expression

$$
\Phi(x)=\int_{L} \frac{n_{o} n_{e} d l}{\sqrt{n_{o}^{2} \sin ^{2} \theta(x, l)+n_{e}^{2} \cos ^{2} \theta(x, l)}} .
$$

E-mail: serva@ifm.liu.se
Here $L$ is the trajectory of the light beam, $n_{o}, n_{e}$ are the principal refractive indexes, and $\theta$ is the angle between the wave vector and the optical axis (director).

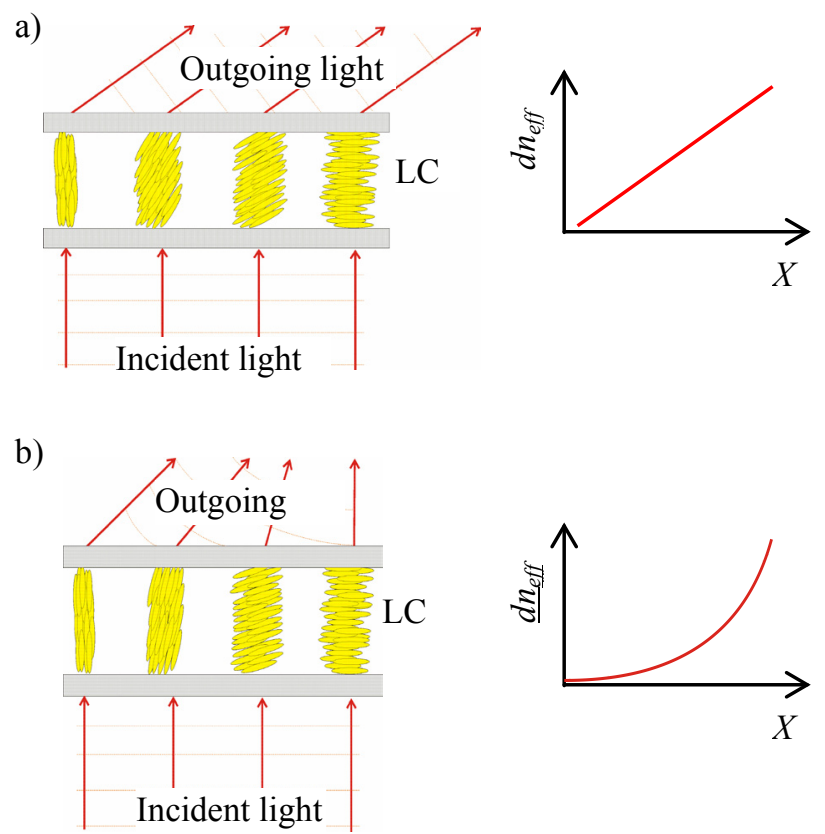

Fig. 1. LC optical elements and their optical paths $\left(d n_{\text {eff }}\right)$ vs. the space coordinate $X$ : a) beam steering device; b) lens.

In order to obtain a beam steering device (Fig.1 a), the optical path has to be varied along the spatial coordinate $x$ as:

$$
\Phi(x)=\Phi_{o}+\alpha x
$$

where $\Phi_{o}$ and $\alpha$ are the coefficients.

The steering angle in this case is defined as $\tan ^{-1} \alpha$. Evidently, the steering angle reaches its maximal value when the effective refractive index varies between $n_{o}$ and $n_{e}$. Performing simple mathematical transformations, it is possible to derive the maximal value of $\alpha$ that is $\frac{\Delta n d}{D}$, where $\Delta n$ is birefringence of the LC, $d$ is the cell gap, and $D$ is the diameter of the light beam. 
Let us evaluate the range of maximal steering angle and focal distance of transmissive optical elements shown in Fig. 1. If we assume that $\Delta n$ can be more than $0.5[1,2]$ and the ratio $d / D$ is around 0.1 , then the steering angle can be controlled within the range $\pm 3^{\circ}$; for $d / D=1$, the range of the steering angle control can be expanded up to $\pm 27^{\circ}$.

The optical path of a lens should satisfy the expression:

$$
\Phi(x)=\Phi_{o}+\beta x^{2} .
$$

Here $\beta$ is a coefficient. Assuming that $\Phi_{o}=n_{o} d$ and $\Phi(R)=n_{e} d$, where $R$ is the lens radius, we derive:

$$
\beta=\frac{\Delta n d}{R^{2}} .
$$

From the information presented above follows that the efficiency of LC element increases with increasing the retardation $\Delta$ nd and with decreasing the diameter $D$ (or $R$ ). However, the retardation cannot be as large as it is required for many prisms and lenses, because of the restriction on the cell gap value. When $d$ is large, e.g. more than $100 \mu \mathrm{m}$, the alignment layers do not have sufficient influence on the LC director distribution. In addition to difficulties associated with formation of a desired director distribution, the value of the cell gap $d$ affects the switching times. Durations of reaction and relaxation are proportional to $d^{2}$ [3].

In order to increase the steering angle and obtain a beam steering device operating with light beams of large diameters, it is necessary to build up optical elements based on the principles of diffractive optics [4]. In this case the needed effect is achieved due to a structure having a broken phase profile (Fig. 2).

Such an approach enables one to use optical elements with the cell gap slightly more than $\frac{\lambda}{\Delta n}$, where $\lambda$ is the wavelength. For $\Delta n$ being around 0.3 and $\lambda=1.55 \mu \mathrm{m}$ the cell gap $d$ has to be around $5 \mu \mathrm{m}$.

The range of the steering angles, as well as the range of the focal distance variation of an LC lens, depends on the minimal period that can be achieved in the diffractive element. The maximal steering angle for normal incident light is defined as $\vartheta=\tan ^{-1} \frac{\lambda}{\Lambda}$, where $\lambda$ is the wavelength, $\Lambda$ is the grating period. Applying the principle of Huygens-Fresnel [5], it is possible to evaluate the minimal distance between two peaks $\Delta x$ :

$$
\Delta x \approx R-\sqrt{R^{2}-2 \lambda f},
$$

where $f$ is the focal distance.
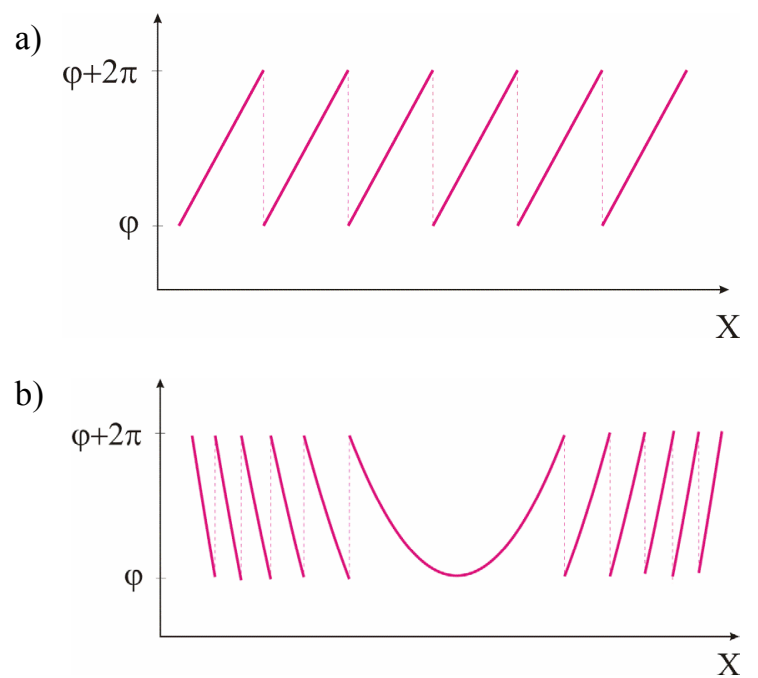

Fig.2. Diffractive optical elements have broken phase profiles. a) beam steering device $b$ ) lens.

On the other hand, $\Delta x$, as well as $\Lambda$, cannot be less than the fly-back zone [6-8], i.e. the minimal transition region in which the LC director changes its orientation. Therefore, the functional abilities of the LC diffractive optical elements are restricted by the fly-back zone.

The authors of the majority of papers dealing with the flyback zone consider this phenomenon as a result of the fringing-field effect [6-8]. However, the calculation carried out by us recently [9] for an LC controlled with uniform electric field in an LC cell that has inhomogeneous anchoring energy gave us the same order of the fly-back zone as for non-uniform electric fields [68]. This fact enables one to claim that the fly-back zone is determined by the elastic properties of the LC material rather than by the fringing-field effect.

The diffraction efficiency of a real diffractive grating can be estimated by the following relationship [7]:

$$
\eta=\eta_{o}(1-\Delta X / \Lambda)^{2}
$$

Here $\eta_{o}$ is the diffraction efficiency of the ideal diffractive grating, and $\Delta X$ is the value of the fly-back zone. $\Delta X$ is the same order as the cell gap $d$.

The ranges of the variations of the steering angle and the variable focal distance can be extended up to two times, if we utilize the reflective mode (Fig. 3) of the LC elements. In this case, the light passes double distance in the LC layer. 


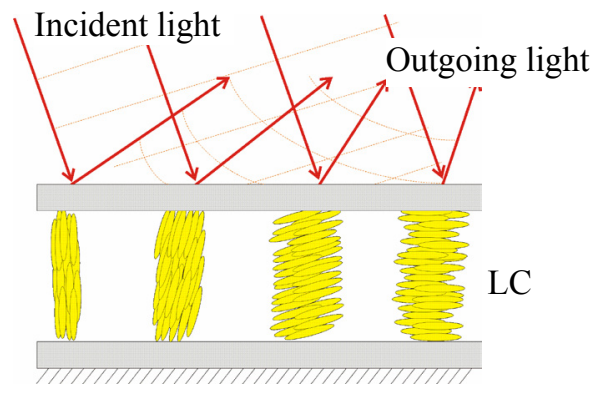

Fig. 3. Reflective LC lens.

Let us evaluate the range of the steering angles and focusing distances that can be achieved with a reflective LC element for the wavelength $1.55 \mu \mathrm{m}$. Let us assume that the birefringence $\Delta n$ can reach $0.5[1,2]$, the fly-back zone is around $d$, and the minimal period is in two times larger than the fly-back zone. Under these assumptions, according to the expression for the maximal steering angle we have $d=1.55 \mu \mathrm{m}$ and the steering angle can be varied within the range $\pm 8.5^{\circ}$. Substituting in Eq. 5 $\Delta x=2 d$, we obtain: $1 / f \approx \pm 5$ diopter for $\mathrm{R}=1 \mathrm{~cm}$, and $1 / f \approx$ \pm 507 diopter for $\mathrm{R}=100 \mu \mathrm{m}$.

The precision to form an electric field distribution depends on the number of electrodes involved, which, in turn, is defined by the size of electrodes and gaps between them. On the other hand, a large number of electrodes involved in forming a period of the phase profile are associated with a large scale of integration and complexity of the driving scheme. In addition, the patterned structure of the electrodes causes undesired diffraction effects.

The difficulties related to the patterned electrodes structure can be overcome by exploiting the approaches based on inhomogeneous boundary conditions, i.e. inhomogeneous anchoring [9-11] or pretilt angle [12]. In this case, the threshold voltage and, as a result, the director distribution are functions of the spatial coordinate, along which the anchoring energy or the pretilt angle are varied. By adjusting the boundary conditions, it is possible to achieve the needed distribution of the LC director.

LC optical elements with inhomogeneous anchoring energy do not affect the light propagation when the voltage is off. The value of the driving voltage is around the threshold voltage of the LC with strong anchoring and saturation voltage of the LC with weak anchoring $[9,10]$. Quite opposite, LC optical elements based on inhomogeneous pretilt angle change the light waveform when the voltage is off, and have no effect on light propagation under strong voltage applied.
The advantages of the approaches based on inhomogeneous boundary conditions are the following: 1) the LC element can have only two single electrodes, 2) it is possible to achieve a relatively high resolution in an $\mathrm{LC}$ diffractive optical element. In fact, the period of the diffraction grating can be around several micrometers. 3) The driving voltage of the approach based on inhomogeneous anchoring is less than the voltages usually used for LC devices. On the other hand, multielectrode structures enable one to generate different intricate distributions of the $\mathrm{LC}$ director.

In acknowledgments, the work has been supported by the Swedish Research Council Formas and HKUST grants CERG 612310 and CERG 612409.

\section{References}

[1] R. Dabrowski, J. Dziaduszek, A. Ziolek, L. Szczucinski, Z. Stolarz, G. Sasnouski, V. Bezborodov, W. Lapanik, S. Gauza, S. T. Wu, OptoElectr. Rev. 15, 47 (2009).

[2] S. Gauza, C.H. Wen, S.T. Wu, N. Janarthanan, C.S. Hsu, Jpn. J. Appl. Phys. 43, 7634 (2004).

[3] V.G. Chigrinov, Liquid Crystal Devices: Physics and Applications (Artech House, Norwood, MA, 1999).

[4] B. Kress, P. Meyrueis: Digital Diffractive Optics (Wiley, Chicester 2000).

[5] M. Born, E. Wolf, Principles of Optics (Cambridge U. Press, 1999)

[6] B. Apter, U. Efron, E. Bahat-Treidel, Appl. Opt. 43, 11 (2004).

[7] P.F. McManamon, T.A. Dorschner, D.L. Corkum, L.J.Friedman, D.S. Hobbs, M. Holz, S. Liberman, H.Q. Nguyen, D.P. Resler, R.C. Sharp, E.A. Watson, Proc. IEEE 84, 268 (1996).

[8] P.F. McManamon, P.J. Bos, M.J. Escuti, J. Heikenfeld, S. Serati, H. Xie, E.A. Watson, Proc. IEEE 97, 1078 (2009).

[9] S. Valyukh, I. Valyukh, V. Chigrinov, H. S. Kwok, H. Arwin, Appl Phys. Lett. 97, 231120 (2010)

[10] S. Valyukh, V. Chigrinov, H.S. Kwok, Digest SID 08, 659 (2008).

[11] A.A. Muravsky, V.E. Agabekov, A.L. Tolstik, U.V. Mahilny, Semicon. Phys. Quant. Electr. Optoel. 13, 154 (2010)

[12] M. Ye, Y. Yokoyama, S. Sato, Appl. Phys. Lett. 89, 141112 (2006).

[13] V. Chigrinov, H.S. Kwok, H. Takada, H. Takatsu, Liq. Cryst. Today 14 1, (2005). 